\title{
Risk factors for reinsertion of urinary catheter after early removal in thoracic surgical patients
}

John Young, MD, ${ }^{\mathrm{a}}$ Travis Geraci, MD, ${ }^{\mathrm{a}}$ Steven Milman, MD, FACS, ${ }^{\mathrm{a}}$ Andrew Maslow, MD,

Richard N. Jones, $\mathrm{ScD},{ }^{\mathrm{c}}$ and Thomas $\mathrm{Ng}, \mathrm{MD}, \mathrm{FACS}^{\mathrm{a}}$

\section{ABSTRACT}

Objectives: To reduce the incidence of urinary tract infection, Surgical Care Improvement Project 9 mandates the removal of urinary catheters within 48 hours postoperatively. In patients with thoracic epidural anesthesia, we sought to determine the rate of catheter reinsertion, the complications of reinsertion, and the factors associated with reinsertion.

Methods: We conducted a prospective observational study of consecutive patients undergoing major pulmonary or esophageal resection with thoracic epidural analgesia over a 2-year period. As per Surgical Care Improvement Project 9, all urinary catheters were removed within 48 hours postoperatively. Excluded were patients with chronic indwelling catheter, patients with urostomy, and patients requiring continued strict urine output monitoring. Multivariable logistic regression analysis was used to identify independent risk factors for urinary catheter reinsertion.

Results: Thirteen patients met exclusion criteria. Of the 275 patients evaluated, $60(21.8 \%)$ required reinsertion of urinary catheter. There was no difference in the urinary tract infection rate between patients requiring reinsertion $(1 / 60$ $[1.7 \%])$ versus patients not requiring reinsertion $(1 / 215[0.5 \%], P=.389)$. Urethral trauma during reinsertion was seen in 1 of 60 patients $(1.7 \%)$. After reinsertion, discharge with urinary catheter was required in 4 of 60 patients $(6.7 \%)$. Multivariable logistic regression analysis found esophagectomy, lower body mass index, and benign prostatic hypertrophy to be independent risk factors associated with catheter reinsertion after early removal in the presence of thoracic epidural analgesia.

Conclusions: When applying Surgical Care Improvement Project 9 to patients undergoing thoracic procedures with thoracic epidural analgesia, consideration to delayed removal of urinary catheter may be warranted in patients with multiple risk factors for reinsertion. (J Thorac Cardiovasc Surg 2018;156:430-5)

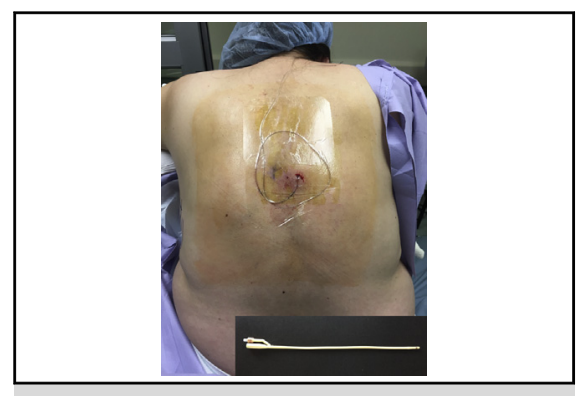

Thoracic surgical patient with thoracic epidural analgesia and urinary catheter.

\section{Central Message}

Because of the risk for urinary retention, delayed removal of a urinary catheter should be considered in patients undergoing thoracic surgery with thoracic epidural analgesia who have multiple risk factors.

\section{Perspective}

Early removal of urinary catheters after surgery is aimed at decreasing the risk of UTI. Patients with thoracic epidural analgesia may have a higher risk of urinary retention. Assessing the incidence and risk factors of urinary retention after early catheter removal in this patient population will help identify those patients in whom delayed catheter removal should be considered.

See Editorial Commentary page 436.
The Surgical Care Improvement Project (SCIP) is an initiative by The Centers for Medicare and Medicaid Services and The Joint Commission to decrease the rates of surgical complications. ${ }^{1}$ SCIP 9 mandates the removal of urinary catheters within 48 hours after surgery to reduce the risk

From the Departments of ${ }^{\text {a }}$ Surgery, ${ }^{\mathrm{b}}$ Anesthesiology, and ${ }^{\mathrm{c}}$ Psychiatry and Human Behavior, The Warren Alpert Medical School of Brown University, Providence, RI.

Received for publication July 18, 2017; revisions received Jan 28, 2018; accepted for publication Feb 7, 2018; available ahead of print March 30, 2018.

Address for reprints: Thomas Ng, MD, FACS, 2 Dudley St, Suite 470, Providence, RI 02905 (E-mail: tng@usasurg.org).

$0022-5223 / \$ 36.00$

Copyright (c) 2018 by The American Association for Thoracic Surgery

https://doi.org/10.1016/j.jtcvs.2018.02.076 of urinary tract infections (UTIs) from a prolonged indwelling foreign body in the urinary bladder. ${ }^{1,2}$

Patients with thoracic epidural analgesia are not exempted from SCIP 9. However, thoracic epidural analgesia may increase the rates of urinary retention after urinary catheter removal, with studies reporting rates as high as $20 \%$. $^{3-5}$ This may lead to the need for re-catheterization and the

$>$ Scanning this QR code will take you to a supplemental video for the article. 

Abbreviations and Acronyms
$\mathrm{BMI}=$ body mass index
$\mathrm{BPH}=$ benign prostatic hypertrophy
SCIP $=$ Surgical Care Improvement Project
$\mathrm{UTI}=$ urinary tract infection

discomfort and complications that may arise from this, which can potentially offset the benefits of early catheter removal. In this study, we sought to determine the outcomes of applying SCIP 9 to thoracic surgical patients, which includes identifying the incidence of re-catheterization, UTI, and urethral trauma, and in addition identify factors associated with the need for catheter reinsertion after early removal in this patient population.

\section{MATERIALS AND METHODS}

Over a 2-year period, from 2013 to 2015, patients aged more than 18 years undergoing major pulmonary or esophageal resection with thoracic epidural analgesia were entered into a prospective observational study. Patients undergoing thoracoscopic procedures were not evaluated, because our institution does not routinely use thoracic epidural analgesia for this patient population. Thoracic epidural catheters were placed preoperatively by staff anesthesiologists at the T5/6 level for both pulmonary and esophageal resections. During the surgical procedure, 1 to $4 \mathrm{mg}$ of morphine and $5 \mathrm{~mL}$ of $0.125 \%$ bupivacaine were administered via the epidural catheter. In the postoperative period, patients received an epidural infusion mixture of hydromorphone $20 \mu \mathrm{g} / \mathrm{mL}$ with bupivacaine $0.06 \%$ in normal saline at a rate of 6 to $10 \mathrm{~mL} / \mathrm{h}$. To keep the infusion constant throughout the study, rather than removing 1 component of the epidural infusion mixture (local anesthesia or narcotic), intravenous infusion of phenylephrine was used for hypotension and intravenous infusion of low-dose naloxone was used for somnolence. Epidural catheters were maintained until the removal of the patient's chest tube or removed on postoperative day 5 regardless of the presence of the chest tube.

We excluded patients with a chronic indwelling catheter, patients with a urostomy, and patients who needed continued strict urine output monitoring beyond 48 hours as determined by the surgical team, because they would not fall under the SCIP mandate. Otherwise, all patients, within 48 hours after their surgical procedure, had their urinary catheters removed in concordance with SCIP mandates. Patients who failed their trial of voiding, defined as 8 hours duration and with more than $500 \mathrm{~mL}$ on bladder scan per institutional nursing protocol, ${ }^{6}$ had their urinary catheter reinserted and removal would then occur 6 hours after discontinuation of the epidural catheter. Consort flow diagram for this study is shown in Figure 1.

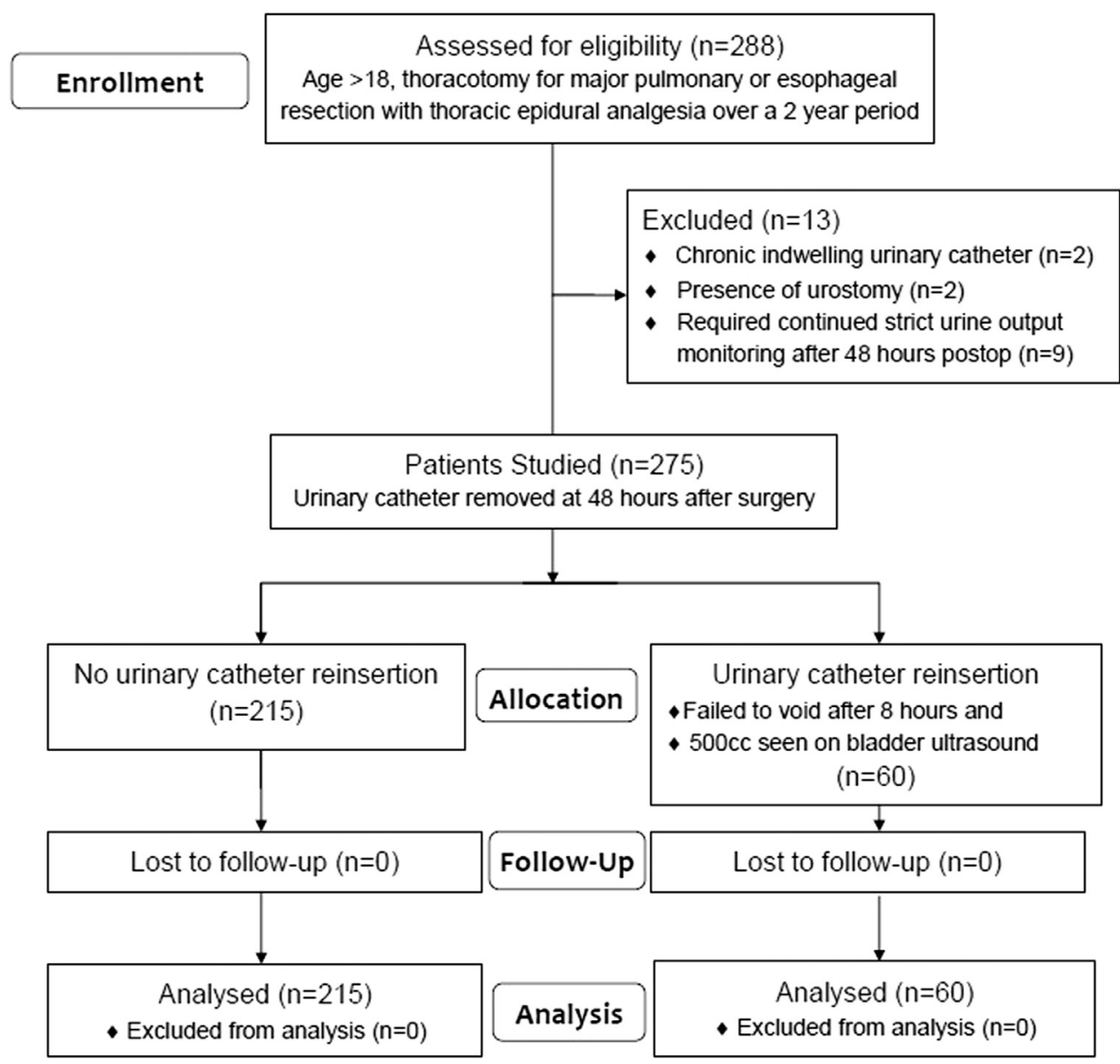

FIGURE 1. Consort flow diagram. 
Outcomes evaluated were the rate of urinary catheter reinsertion, complications of reinsertion, UTI rate (defined as $\geq 10^{5}$ colony forming units per milliliter on culture), and the rate of discharge with an indwelling urinary catheter. Factors associated with catheter reinsertion were also evaluated. Categoric data were presented as counts with the corresponding percentages and compared using the chi-square test or Fisher exact test where appropriate. Continuous data were presented as medians with interquartile range and compared using Mann-Whitney $U$ test or Student $t$ test where appropriate. Multivariable logistic regression analysis was used to assess for independent risk factors associated with urinary catheter reinsertion, force entering variables with a $P$ value less than .1 found at univariate analysis. To evaluate for multicollinearity, variance inflation factor was calculated for each variable, the results of which were also used to determine the final multivariable model.

Our Institutional Review Board approved this prospective study (ID750253-2) and waived patient consent because this was an observational study and SCIP 9, the early removal of urinary catheters postoperatively, was a quality-improvement initiative mandated by The Centers for Medicare and Medicaid Services and The Joint Commission during the time of this study.

\section{RESULTS}

During the study time period, 288 consecutive patients underwent major thoracic surgical procedure with the use of thoracic epidural analgesia. Thirteen patients met exclusion criteria: 2 with a chronic indwelling urinary catheter, 2 with a urostomy, and 9 requiring continued strict urine output monitoring after 48 hours. Therefore, 275 patients were evaluated, with 215 patients undergoing pulmonary resection by thoracotomy and 60 patients undergoing open esophageal resection, all receiving thoracic epidural analgesia. Patient demographics, details of the surgical procedure, and postoperative mortality are displayed in Table 1. For patients undergoing pulmonary resection, there was no difference in the rate of catheter reinsertion for segmentectomy $(4 / 21[19 \%])$, lobectomy $(28 / 183[15 \%])$, and pneumonectomy $(1 / 11[9 \%])(P=.758)$. For patients undergoing esophageal resection, there was no difference in the rate of catheter reinsertion for transhiatal approach $(5 / 8[62 \%])$ and transthoracic approach $(22 / 52[42 \%])$ $(P=.448)$.

For the entire study population of 275 patients, 60 $(21.8 \%)$ required reinsertion of a urinary catheter after early removal in the presence of thoracic epidural analgesia, and the overall UTI rate was $0.7 \%(2 / 275)$. To provide a general comparison, the UTI rate 2 years before implementing this study of SCIP 9, from 2010 to 2012 in the same patient population of major pulmonary or esophageal resection with thoracic epidural analgesia, was $1.9 \%$ $(5 / 267)(P=.279)$. Unadjusted comparison of outcomes between patients requiring urinary catheter reinsertion versus patients not requiring urinary catheter reinsertion is shown in Table 2. There was no difference in the rate of UTI between the patients requiring catheter reinsertion $(1 / 60$ $[1.7 \%])$ versus patients not requiring reinsertion $(1 / 215$

TABLE 1. Patient demographics

\begin{tabular}{|c|c|c|c|}
\hline Characteristic* & Pulmonary resection $(n=215)$ & Esophageal resection $(n=60)$ & All patients $(n=275)$ \\
\hline Age, y & $65(57-72)$ & $64(53-72)$ & $65(57-72)$ \\
\hline \multicolumn{4}{|l|}{ Gender } \\
\hline Female & $127(59.1 \%)$ & $14(23.3 \%)$ & $141(51.3 \%)$ \\
\hline Male & $88(40.9 \%)$ & $46(76.7 \%)$ & $134(48.7 \%)$ \\
\hline BMI kg/m² & $26.9(24.0-31.6)$ & $26.8(24.0-29.6)$ & $26.9(24.0-31.1)$ \\
\hline Charlson Comorbidity Index & $1(0-2)$ & $1(0-2)$ & $1(0-2)$ \\
\hline Male gender with BPH & $19 / 88(21.6 \%)$ & $5 / 46(10.9 \%)$ & $24 / 134(17.9 \%)$ \\
\hline \multicolumn{4}{|l|}{ Pulmonary } \\
\hline Segmentectomy & $21(9.8 \%)$ & & \\
\hline Lobectomy & $183(85.1 \%)$ & & \\
\hline Pneumonectomy & $11(5.1 \%)$ & & \\
\hline \multicolumn{4}{|l|}{ Esophageal } \\
\hline Transhiatal & & $8(13.3 \%)$ & \\
\hline Transthoracic $\dagger$ & & $52(86.7 \%)$ & \\
\hline Operative time, $\min$ & $100(85-125)$ & $282(260-315)$ & $115(90-200)$ \\
\hline Urinary catheter reinsertion & $33(15.3 \%)$ & $27(45.0 \%)$ & $60(21.8 \%)$ \\
\hline Epidural, d & $3(2-4)$ & $5(5-6)$ & $3(2-5)$ \\
\hline Chest tube, $\mathrm{d}$ & $3(2-4)$ & $5(5-6)$ & $3(2-5)$ \\
\hline Hospital, d & $5(4-6)$ & $10(9-14)$ & $5(4-9)$ \\
\hline Mortality (30 d or in hospital) & 0 & $1(1.7 \%)$ & $1(0.4 \%)$ \\
\hline
\end{tabular}

BMI, Body mass index; $B P H$, benign prostatic hypertrophy. ${ }^{*}$ Continuous variables expressed as median with interquartile range. $\dagger 46$ Ivor Lewis, 3 three incision, 3 left thoracoabdominal. 
TABLE 2. Unadjusted outcomes, urinary catheter reinsertion versus no reinsertion

\begin{tabular}{|c|c|c|c|}
\hline Outcomes* & $\begin{array}{l}\text { No reinsertion } \\
\quad(\mathbf{n}=\mathbf{2 1 5})\end{array}$ & $\begin{array}{l}\text { Reinsertion } \\
\quad(\mathbf{n}=\mathbf{6 0})\end{array}$ & $\begin{array}{c}P \\
\text { value }\end{array}$ \\
\hline Urinary catheter, $\mathrm{d}$ & $2(2-2)$ & $5(4-6)$ & $<.001$ \\
\hline Catheter reinsertion trauma & & $1(1.7 \%)$ & \\
\hline UTI & $1(0.5 \%)$ & $1(1.7 \%)$ & .389 \\
\hline Epidural, d & $3(2-5)$ & $4.5(3-5)$ & $<.001$ \\
\hline Chest tube, $\mathrm{d}$ & $3(2-5)$ & $4.5(3-6)$ & $<.001$ \\
\hline Hospital, d & $5(4-7)$ & $8.5(5-12)$ & $<.001$ \\
\hline $\begin{array}{l}\text { Discharged with urinary } \\
\text { catheter }\end{array}$ & & $4(6.7 \%) \dagger$ & \\
\hline
\end{tabular}

[0.5\%], $P=.389$ ). Of the 60 patients requiring urinary catheter reinsertion, $1(1.7 \%)$ had traumatic reinsertion with hematuria that ultimately resolved without sequelae, and because of a repeat episode of urinary retention, 4 $(6.7 \%)$ were discharged with an indwelling urinary catheter requiring urology follow-up for outpatient management.

Univariate analysis for factors associated with urinary catheter reinsertion after applying SCIP 9 to patients with thoracic epidural analgesia is shown in Table 3. Older age, male gender, lower body mass index (BMI), benign prostatic hypertrophy $(\mathrm{BPH})$, longer operative time, and esophagectomy were found to be significant risk factors. After multivariable logistic regression analysis, esophagectomy, lower BMI, and BPH remained independent factors associated with urinary catheter reinsertion after early removal in patients with thoracic epidural analgesia
(Table 4). Evaluation for multicollinearity found a high correlation between esophagectomy and operative time (variance inflation factor of 3.67); therefore, operative time was excluded from the final multivariable model seen in Table 4.

\section{DISCUSSION}

The removal of urinary catheters within 48 hours after operation as mandated by SCIP 9 is aimed at decreasing the rates of catheter-associated UTI, citing data that show a 2-fold increased risk of UTI with catheterization duration greater than 2 days in postoperative cardiac, colorectal, vascular, and orthopedic patients. ${ }^{1,2}$ These data do not specifically address thoracic patients with thoracic epidural analgesia, because early removal of urinary catheters in this patient population may lead to urinary retention resulting in re-catheterization and its potential complications. $^{3-5}$ Therefore, the proposed benefits of SCIP 9 become less clear.

Three randomized trials have been published in an attempt to address this question, but with conflicting results. Zaouter and colleagues ${ }^{7}$ evaluated 215 patients at low risk for urinary retention (excluding patients with $\mathrm{BPH}$ ) undergoing thoracic or abdominal procedures with thoracic epidural analgesia, randomizing patients to urinary catheter removal on postoperative day 1 versus removal after discontinuation of the epidural catheter. In this study, the rate of recatheterization trended higher in the early removal group but did not reach statistical significance perhaps because of the lack of power, $7.6 \%$ versus $1.2 \%(P=.09)$; however, the rate of UTI was lower in the early catheter removal group, $2 \%$ versus $14 \%(P=.004)$. On the basis of these

TABLE 3. Univariate analysis for factors associated with urinary catheter reinsertion

\begin{tabular}{|c|c|c|c|c|}
\hline Characteristic* & No reinsertion $(n=215)$ & Reinsertion $(n=60)$ & $P$ value & Crude OR $(95 \%$ CI $)$ \\
\hline Age, y & $64(56-71)$ & $68(60-74)$ & .048 & $1.8(1.0-3.2)$ \\
\hline \multicolumn{5}{|l|}{ Gender } \\
\hline Female & $124 / 141(87.9 \%)$ & $17 / 141(12.1 \%)$ & $<.001$ & $0.3(0.2-0.5)$ \\
\hline Male & $91 / 134(67.9 \%)$ & $43 / 134(32.1 \%)$ & & \\
\hline BMI kg/m² & $27.3(24.0-32.1)$ & $26.0(23.3-28.8)$ & .021 & $0.4(0.2-0.9)$ \\
\hline \multicolumn{5}{|l|}{ Diabetes } \\
\hline Yes & $43 / 55(78.2 \%)$ & $12 / 55(21.8 \%)$ & 1.000 & \\
\hline No & $172 / 220(78.2 \%)$ & $48 / 220(21.8 \%)$ & & \\
\hline Charlson Comorbidity Index & $1(0-2)$ & $1(0-2)$ & .127 & \\
\hline \multicolumn{5}{|l|}{ Male gender } \\
\hline With BPH & $12 / 24(50.0 \%)$ & $12 / 24(50.0 \%)$ & .038 & $2.5(1.0-6.3)$ \\
\hline Without BPH & $79 / 110(71.8 \%)$ & $31 / 110(28.2 \%)$ & & \\
\hline \multicolumn{5}{|l|}{ Procedure } \\
\hline Pulmonary & $182 / 215(84.7 \%)$ & $33 / 215(15.3 \%)$ & $<.001$ & \\
\hline Esophageal & $33 / 60(55.0 \%)$ & $27 / 60(45.0 \%)$ & & $4.5(2.4-8.5)$ \\
\hline Operative time, $\min$ & $110(90-65)$ & $155(90-270)$ & .005 & $2.5(1.5-4.3)$ \\
\hline
\end{tabular}

$O R$, Odds ratio; $C I$, confidence interval; $B M I$, body mass index; $B P H$, benign prostatic hypertrophy. ${ }^{*}$ Continuous variables expressed as median with interquartile range. 
TABLE 4. Multivariable logistic regression analysis for factors associated with urinary catheter reinsertion

\begin{tabular}{lcc}
\hline \multicolumn{1}{c}{ Factors } & $\boldsymbol{P}$ value & OR $(\mathbf{9 5} \% \mathbf{C I})$ \\
\hline Esophageal resection (vs pulmonary) & $<.001$ & $4.16(2.05-8.43)$ \\
Presence of $\mathrm{BPH}^{*}$ & & \\
$\quad$ Female & .056 & $0.49(0.24-1.02)$ \\
Male without BPH & reference & \\
$\quad$ Male with BPH & .049 & $2.73(1.00-7.45)$ \\
BMI & .029 & $0.93(0.87-0.99)$ \\
Age & .070 & $1.03(0.99-1.06)$ \\
\hline
\end{tabular}

$O R$, Odds ratio; $C I$, confidence interval; $B P H$, benign prostatic hypertrophy; $B M I$, body mass index. *BPH variable includes men with $\mathrm{BPH}$, men without $\mathrm{BPH}$ and women, combined

findings, Zaouter and colleagues ${ }^{7}$ recommended early removal of urinary catheters in this patient population. Chia and colleagues ${ }^{8}$ randomized 78 patients undergoing thoracic procedures with thoracic epidural analgesia to early urinary catheter removal on postoperative day 1 versus removal after the discontinuation of the epidural catheter, finding no instances of urinary retention or UTI in either group. Chia and colleagues ${ }^{8}$ also recommended early urinary catheter removal. In a more recent study by Allen and colleagues, ${ }^{5} 247$ patients undergoing thoracic procedures with thoracic epidural analgesia were randomized to urinary catheter removal within 48 hours versus removal after discontinuation of the epidural catheter. In this study, the rate of re-catheterization was higher in the early removal group, $12.4 \%$ versus $3.2 \%(P=.006)$, but there was no difference in the rate of UTI between the early versus delayed catheter removal groups, $0 \%$ versus $0.8 \%$, respectively $(P=.49)$. Thus, Allen and colleagues ${ }^{5}$ recommended removal of the urinary catheter after discontinuation of the epidural catheter.

Although there remains uncertainty regarding the optimal timing of urinary catheter removal in thoracic surgical patients with thoracic epidural analgesia, we think in principle that there is merit to early removal of urinary catheters in terms of reducing the risk of infection, patient comfort, and early patient mobilization. However, this must be balanced against urinary retention that can lead to the discomfort of catheter reinsertion and the complications of re-catheterization. The goal of this study was 2-fold: to evaluate the rate of re-catheterization and its related risk of complications and to evaluate the risk factors associated with retention and re-catheterization, hoping to provide data to guide thoracic surgeons in selecting patients for delayed catheter removal. We found that the rate of catheter reinsertion after early removal was $21.8 \%$, which is consistent with the literature for this patient population. ${ }^{4,5}$ Catheter reinsertion was not without complication because 1 patient $(1.7 \%)$ had hematuria/ureteral trauma. Catheter reinsertion did not result in a higher rate of UTI versus those not requiring reinsertion, $1.7 \%$ versus $0.5 \%$, respectively $(P=.389)$. This lack of statistical significance may be due to the limited number of patients enrolled, reflecting inadequate statistical power. As our data illustrate, urinary retention and re-catheterization are not benign processes. Ideally, decision-making for catheter removal in the presence of thoracic epidural analgesia would be guided by a patient's risk profile. We evaluated independent risk factors associated with urinary retention after early catheter removal by multivariate analysis, finding esophagectomy, BPH, and lower BMI to be significant. It is not surprising that male gender with BPH is a risk factor, and perhaps also not unexpected is our finding that esophagectomy was an independent risk factor. Because this procedure is more extensive than pulmonary resection, we hypothesize that patients with esophagectomy are likely to have more pain due to multiple incisions and are likely to receive a larger volume of intravenous fluid intraoperatively and postoperatively. Given our results, for patients undergoing esophagectomy or with a history of BPH, our current practice now delays the removal urinary catheters until after the removal of epidural catheters. For all other patient populations, urinary catheters are removed at 48 hours after surgery. Our analysis found lower BMI to be an independent risk factor, a result we cannot clearly explain, and further study is needed to clarify this association.

\section{Study Limitations}

Our study has several limitations. First, this is not a randomized controlled trial. However, our aim was not to compare early versus delayed urinary catheter removal in this patient population, because randomized studies regarding this have been published, as discussed earlier. ${ }^{5,7,8}$ Our institution agrees with the goal of early urinary catheter removal and its proposed benefits; therefore, we restricted our study to the early catheter removal cohort as per SCIP 9. Because re-catheterization can result in its own set of complications, we sought to evaluate risk factors associated with urinary retention to guide thoracic surgeons in selectively delaying catheter removal in high-risk patients. Second, our study lacks data regarding pain scores and the amount of rescue narcotic use, which may be helpful in assessing the effectiveness and thus the successful positioning of the thoracic epidural catheter for each patient. Third, the small number of patients is another limitation of our study, which limits the power of our analysis, especially when it comes to stratifying for specific patient populations within our cohort. Further study with a larger number of patients is needed to confirm our results and to possibly create useful clinical score models that provide more accurate and objective measures regarding the probability of urinary retention after early catheter removal for any given combination of patient characteristics. Finally, our data certainly do not 


\section{Risk Factors for Reinsertion of Urinary Catheter Following Early Removal in Thoracic Surgical Patients}

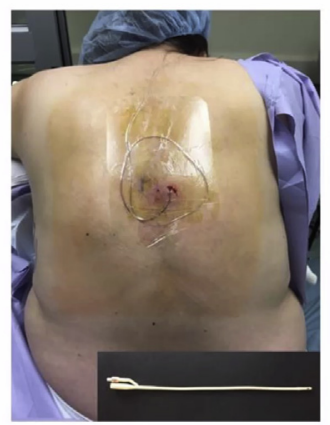

John Young, Travis Geraci, Steven Milman, Andrew Maslow, Richard Jones, and Thomas Ng

The Warren Alpert Medical School of Brown University Providence, RI

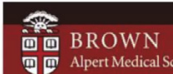

VIDEO 1. Risk factors for reinsertion of urinary catheter after early removal in thoracic surgical patients. Video available at: http://www. jtcvsonline.org/article/S0022-5223(18)30617-2/fulltext.

apply to thoracoscopic approaches performed without thoracic epidural analgesia; however, open approaches by thoracotomy will always be part of the armamentarium of thoracic surgeons, and our data should continue to be relevant.

\section{CONCLUSIONS}

Early urinary catheter removal in surgical patients aimed at lowering the risk of catheter-associated UTI seems appropriate. However, thoracic surgical patients represent a unique population because the presence of thoracic epidural analgesia may increase the rate of urinary retention, resulting in the discomfort of re-catheterization and its potential complications. Our study identifies several independent risk factors associated with urinary retention in this patient population, that being esophagectomy, presence of $\mathrm{BPH}$, and low BMI (Video 1); therefore, consideration should be given to delaying the removal of urinary catheters in patients with these risk factors.

\section{Conflict of Interest Statement}

Authors have nothing to disclose with regard to commercial support.

\section{References}

1. Centers for Medicare \& Medicaid Services/The Joint Commission. CMS hospital compare. SCIP-Inf-9: urinary catheter removed on postoperative day 1 or 2 with day of surgery being day zero. Available at: http://www.hospitalsafetyscore.org/ media/file/SCIPINF9.pdf. Accessed May 2, 2017

2. Wald HL, Ma A, Bratzler DW, Kramer AM. Indwelling urinary catheter use in the postoperative period: analysis of the national surgical infection prevention project data. Arch Surg. 2008;143:551-7.

3. Wuethrich PY, Burkhard FC. Thoracic epidural analgesia: what about the urinary bladder? Trends Anaesth Crit Care. 2012;2:138-44.

4. Hu Y, Craig SJ, Rowlingson JC, Morton SP, Thomas CJ, Persinger MB, et al. Early removal of urinary catheter after surgery requiring thoracic epidural: a prospective trial. J Cardiothorac Vasc Anesth. 2014;28:1302-6.

5. Allen MS, Blackmon SH, Nichols FC III, Cassivi SD, Harmsen WS, Lechtenberg B, et al. Optimal timing of urinary catheter removal after thoracic operations: a randomized controlled study. Ann Thorac Surg. 2016;102:925-30.

6. Tripepi-Bova KA, Sun Z, Mason D, Albert NM. Early removal of urinary catheters in patients with thoracic epidural catheters. J Nurs Care Qual. 2013;28:340-4.

7. Zaouter C, Kaneva P, Carli F. Less urinary tract infection by earlier removal of bladder catheter in surgical patients receiving thoracic epidural analgesia. Reg Anesth Pain Med. 2009;34:542-8.

8. Chia YY, Wei RJ, Chang HC, Liu K. Optimal duration of urinary catheterization after thoracotomy in patients under postoperative patient-controlled epidural analgesia. Acta Anaesthesiol Taiwan. 2009;47:173-9.

Key Words: pulmonary resection, esophagectomy, thoracotomy, epidural analgesia, urinary catheter, urinary retention 